Pacific Journal of Mathematic 


\title{
A CONDITIONALLY COMPACT POINT SET WITH NONCOMPACT CLOSURE
}

\author{
DAVID E. CoOK
}

\begin{abstract}
Sometime in 1930, Leo Zippin showed that there exists a complete Moore space that contains a conditionally compact point set whose closure is not compact. It is the object of this naper to show that if the hypothesis of the continuum is true then there exists a separable, complete Moore space which contains such a point set and, furthermore, satisfies R. L. Moore's Axioms 2, 3, 4, 5, and 6. Theorem 1, concerning the existence of certain subsets of the Cartesian plane, is fundamental to the construction of this example and its proof consticates a major portion of this paper.
\end{abstract}

A complete Moore space is one satisfying Axioms 0 and 1 of [1]. The terms compact and conditionally compact are as defined in [2] and other definitions and notation are as in [1].

In a Cartesian plane $E$, let $M$ denote the point set to which the point $P$ belongs if and only if each coordinate of $P$ is a positive integer. Let $H$ denote the collection of point sets to which the point set $h$ belongs if and only if the points of $h$ are the points of an infinite sequence $P_{1}, P_{2}, P_{3}, \cdots$ of points of $M$ such that (a) $P_{1}$ is the point $(1,1)$ and (b) for each positive integer $n, P_{n+1}$ is at a distance of 1 from $P_{n}$ and either above it or to the right of it. For each integer $k$, let $T_{k}$ denote a translation in $E$ such that $T_{k}(0,0)=(k,-k)$. For each point set $h$ of $H$ and each point $P$ of $M$, let $A_{h P}$ denote the set to which $X$ belongs if and only if $X$ is a point of $M \cdot T_{k}(h)$ where $k$ is the integer $j$ such that $T_{j}(h)$ contains $P$. For each point set $h$ of $H$, let $G_{h}$ denote the collection to which $g$ belongs if and only if for some point $P$ of $M, g$ is $A_{h P}$.

Lemma 0 . If $h$ is a point set of $H$ and $P$ and $Q$ are two points of $M$, then either $A_{h P}=A_{h Q}$ or $A_{h P}$ and $A_{h Q}$ do not intersect.

Lemma 1. Suppose $h$ and $h^{\prime}$ are two point sets of $H$ such that $h^{\prime}$ does not contain infinitely many points of any point set of $G_{h}$. Then if $g$ and $g^{\prime}$ are two sets of $G_{h}$ and $G_{h}^{\prime}$ respectively, $g$ does not contain infinitely many points of $g^{\prime}$.

Proof. Suppose $P$ is a point of $g \cdot g^{\prime}$. There exist integers $j$ and $k$ such that $T_{j}(P)$ is a point $X$ of $h$ and $T_{k}(P)$ is a point $Y$ of $h^{\prime}$. There exists an integer $i$ such that $T_{i}(X)=Y$. If $P^{\prime}$ is any 
point of $g \cdot g^{\prime}, T_{i}\left(T_{j}\left(P^{\prime}\right)\right)$ is a point of $h^{\prime}$, thus if $g$ contains infinitely many points of $g^{\prime}$, then $h^{\prime}$ contains infinitely many points of the set $T_{i}\left(T_{j}(g)\right)$ of $G_{h}$.

Let $h_{1}\left(h_{2}\right)$ denote the set of all points of $M$ with abscissa (ordinate) 1. Suppose $h$ is a point set of $H, P_{1}, P_{2}, P_{3}, \ldots$ are the points of a point set $g$ of $G_{h}$, and $P$ and $Q$ are two points of $M$ not in $g$. Let $R$ denote the point set to which the point $X$ belongs if and only if $X$ is a point of either (a) the straight line ray with endpoint $P_{1}$ and slope 1 which contains no point of $M-P_{1}$ or (b) the straight line interval from $P_{i}$ to $P_{i+1}$ for some position integer $i$. Then $P$ and $Q$ are said to lie on opposite sides of $g$ or to lie on the same side of $g$ according as $R$ does or does not separate $P$ from $Q$ in the plane.

Lemma 2. Suppose (1) $H^{\prime}$ is a finite subcollection of $H$ such that $(a) h_{1}$ and $h_{2}$ belong to $H^{\prime}$ and $(b)$ if $h$ and $h^{\prime}$ are two point sets of $H^{\prime}$, no point set of $G_{h}$ has infinitely many points in common with any one point set of $G_{h^{\prime}}$, (2) $P$ is a point of $M$, and (3) $K$ is an infinite subset of $M$ such that for no point set $h$ of $H^{\prime}$ does any point set of $G_{h}$ contain infinitely many points of $K$. Then there exists an infinite subset $K^{\prime}$ of $K$ and a point $Q$ of $K^{\prime}$ such that (1) for each point set $h$ of $H^{\prime}$, each point of $K^{\prime}$ lies on the same side of $A_{h P}$ as Q, (2) for each two point sets $h$ and $h^{\prime}$ of $H^{\prime}$, each point of $K^{\prime}$ lies above and to the right of every point of $\left(A_{h P}\right) \cdot\left(A_{h^{\prime} P}\right)$, and (3) if $Q^{\prime}$ is a point of $K^{\prime}$ distinct from $Q$, there exists a point set $h$ of $H$ containing $Q$ and $Q^{\prime}$ such that no point of $h$ between $Q$ and $Q^{\prime}$ lies on the opposite side of $A_{h^{\prime}}$ from $Q$ for any point set $h^{\prime}$ of $H^{\prime}$.

Proof. Let $h_{1}, h_{2}, h_{3}, \cdots, h_{n}$ denote the point sets of the collection $H^{\prime}$. Let $K_{1}$ denote some infinite subset of $K$ such that each point of $K_{1}$ lies to the right of $A_{h_{1} P}$. There exists a sequence $K_{1}, K_{2}, K_{3}, \cdots, K_{n}$ such that $K_{1}$ is an infinite subset of $K$ and for each positive integer $i$ greater than 1 but not greater than $n, K_{i}$ is an infinite subset of $K_{i-1}$ and each two points of $K_{i}$ lie on the same side of $A_{h_{i} P}$.

Let $W$ denote the point set to which the point $w$ belongs if and only if for some two point sets $h$ and $h^{\prime}$ of $H^{\prime}, w$ is a point of $\left(A_{h P}\right) \cdot\left(A_{h^{\prime} P}\right)$. Since no point set of $G_{h}$ has infinitely many points in common with any one point set of $G_{h^{\prime}}$, it follows from Lemma 1 that $W$ is a finite subset of $M$. There exist points $P_{1}$ and $P_{2}$ of $W$ such that no point of $W$ is above $A_{h_{2} P_{2}}$ and no point of $W$ is to the right of $A_{h_{1} P_{1}}$. Let $K_{n}^{\prime}$ denote the set of all points of $K_{n}$ which lie above $A_{h_{2} P_{2}}$ and to the right of $A_{h_{1} P_{1}}$. Since no point set of $G_{h_{1}}+G_{h_{2}}$ contains infinitely many points of $K_{n}, K_{n}^{\prime}$ is an infinite point set. 
Let $Q$ denote some point of $K_{n}^{\prime}$ and let $K^{\prime}$ denote the set whose points are $Q$ and those points of $K_{n}^{\prime}$ which lie both to the right of $A_{h_{1} Q}$ and above $A_{h_{2} Q}$.

Suppose $Q^{\prime}$ is a point of $K^{\prime}$ distinct from $Q . Q^{\prime}$ lies above and to the right of $Q$. Let $Q^{\prime \prime}$ denote the intersection of $A_{h_{1} Q}$ with $A_{h_{2} Q^{\prime}}$. If no point of $M$ between $Q$ and $Q^{\prime \prime}$ belongs to $A_{h^{\prime} P}$ for any point set $h^{\prime}$ of $H^{\prime}$, let $Z$ denote the subset of $M$ whose points are $Q^{\prime \prime}$, the points of $M$, if any, between $Q$ and $Q^{\prime \prime}$, and the points of $M$, if any, between $Q^{\prime \prime}$ and $Q^{\prime}$. If, however, for some point set $h^{\prime}$ of $H^{\prime}$, $A_{h^{\prime} P}$ contains a point between $Q$ and $Q^{\prime \prime}$, let $P^{\prime}$ denote the lowest such point. Since $Q$ is above and to the right of every point of $W$, $h^{\prime}$ is the only point set $h$ of $H^{\prime}$ such that $A_{h P}$ contains $P^{\prime}$. Since $Q^{\prime}$ lies on the same side of $A_{h^{\prime} P}$ as $Q$, some point of $A_{h^{\prime} P}$ belongs to $A_{h_{2} Q^{\prime}}$ and all such points lie to the left of $Q^{\prime}$. Let $P^{\prime \prime}$ denote the right-most point of $\left(A_{h^{\prime} P}\right) \cdot\left(A_{h_{2} Q^{\prime}}\right)$. In this case, let $Z$ denote the subset of $M$ whose points are $P^{\prime}$; the points of $M$, if any, between $Q$ and $P^{\prime}$; the points of $A_{h^{\prime} P}$, if any, between $P^{\prime}$ and $P^{\prime \prime} ; P^{\prime \prime}$; and the points of $M$, if any, between $P^{\prime \prime}$ and $Q^{\prime}$.

There exists point sets $h_{q}$ and $h_{q^{\prime}}$ of $H$ containing $Q$ and $Q^{\prime}$ respectively. There exist positive integers $i$ and $j$ such that $Q$ and $Q^{\prime}$ are respectively the $i^{\text {th }}$ point of $h_{q}$ and the $j^{\text {th }}$ point of $h_{q^{\prime}}$. Let $Z^{\prime}$ denote the point set whose points are the first $i$ points of $h_{q}$. Let $Z^{\prime \prime}$ denote the point set whose points are those points of $h_{q^{\prime}}$ other than the first $j-1$ points. The point set $Z+Z^{\prime}+Z^{\prime \prime}$ is a point set $h$ of $H$ such that no point of $h$ between $Q$ and $Q^{\prime}$ lies on the opposite side of $A_{h^{\prime} P}$ from $Q$ for any point set $h^{\prime}$ of $H^{\prime}$.

Lemma 3. Suppose (1) $H^{\prime}$ is a countable subcollection of $H$ such that (a) $h_{1}$ and $h_{2}$ belong to $H^{\prime}$ and (b) if $h$ and $h^{\prime}$ are two point sets of $H^{\prime}$, no point set of $G_{h}$ has infinitely many points in common with any one point set of $G_{h^{\prime}}$ and (2) $K$ is an infinite subset of $M$ such that for no point set $h$ of $H^{\prime}$ does any point set of $G_{h}$ contain infinitely many points of $K$. Then there exists a point set $h$ of the collection $H$ such that (1) $h$ contains an infinite subset of $K$ and (2) $h$ does not contain infinitely many points of any point set of $G_{h^{\prime}}$ for any point set $h^{\prime}$ of the collection $H^{\prime}$.

Proof. If $H^{\prime}$ is infinite, let $h_{1}, h_{2}, h_{3}, \ldots$ denote the point sets of $H^{\prime}$. If $H^{\prime}$ is finite, let $h_{1}, h_{2}, h_{3}, \cdots, h_{n}$ denote the point sets of $H^{\prime}$ and for each positive integer $i$ greater than $n$, let $h_{i}$ denote the point set $h_{n}$. For each positive integer $i$, let $H_{i}$ denote the collection whose members are the first $i+1$ point sets of the sequence $h_{1}, h_{2}, h_{3}, \cdots$. Let $P_{0}$ denote the point $(1,1)$ and let $K_{0}$ denote the 
point set $K$.

It follows from Lemma 2 that there exist sequences $K_{1}, K_{2}, K_{3}, \ldots$ and $P_{1}, P_{2}, P_{3}, \ldots$ such that for each positive integer $i, K_{i}$ is an infinite subset of $K_{i-1}$ and $P_{i}$ is a point of $K_{i}$ below and to the left of each point of $K_{i}-P_{i}$ such that (1) for each point set $h$ of $H_{i}, K_{i}$ lies on the same side of $A_{h P_{i-1}}$ as $P_{i}$, (2) for each two point sets $h$ and $h^{\prime}$ of $H_{i}$, each point of $K_{i}$ lies above and to the right of every point of $\left(A_{h P_{i-1}}\right) \cdot\left(A_{h^{\prime} P_{i-1}}\right)$, and (3) if $Q_{i}$ is a point of $K_{i}$ distinct from $P_{i}$, there exists a point set $h_{i}\left(Q_{i}\right)$ of the collection $H$ containing $P_{i}$ and $Q_{i}$ such that no point of $h_{i}\left(Q_{i}\right)$ between $P_{i}$ and $Q_{i}$ lies on the opposite side of $A_{h P_{i-1}}$ from $P_{i}$ for any point set $h$ of $H_{i}$.

There exists a sequence $Z_{1}, Z_{2}, Z_{3}, \ldots$ such that (1) for each positive integer $i, Z_{i}$ is a point set whose points are $P_{i-1}, P_{i}$, and those points of $h_{i-1}\left(P_{i}\right)$, where $h_{0}$ is some point set of $H$ containing $P_{0}$ and $P_{1}$, between $P_{i-1}$ and $P_{i}$ and (2) no point of $Z_{i+1}$ between $P_{i}$ and $P_{i+1}$ lies on the opposite side of $A_{h P_{i-1}}$ from $P_{i}$ for any point set $h$ of $H_{i}$.

$Z_{1}+Z_{2}+Z_{3}+\cdots$ is a point set $h$ of the collection $H$ which contains the infinite subset $P_{1}+P_{2}+P_{3}+\cdots$ of $K$.

Suppose $h$ contains infinitely many points of some point set $g$ of $G_{h^{\prime}}$ for some point set $h^{\prime}$ of $H^{\prime}$. For some positive integer $j, h^{\prime}$ is the term $h_{j}$ of the sequence $h_{1}, h_{2}, h_{3}, \ldots A_{h_{j} P_{j}}, A_{h_{j} P_{j+1}}, A_{h_{j} P_{j+2}}, \ldots$ is an infinite sequence such that for each positive integer $i, A_{h_{j} P_{j+i}}$ and $A_{h_{j} P_{j+i+1}}$ lie on the same side of $A_{h_{j} P_{j}}$ and $Z_{j+i+1}$ lies on the same side of $A_{h_{j} P_{j+i-1}}$ as $P_{j+i}$. Thus for each positive integer $i$, only finitely many points of $h$ are on the same side of $A_{h_{j} P_{j+i}}$ as $P_{j-1}$. Therefore, contrary to supposition, no point set of $G_{h_{j}}$ contains infinitely many points of $h$.

Lemma 4. If $H^{\prime}$ is a countable subcollection of $H$, then there exists an infinite subset $K$ of $M$ such that for each point set $h$ of $H^{\prime}$, no point set of $G_{h}$ contains infinitely many points of $K$.

Proof. Let $G$ denote the collection to which $g$ belongs if and only if for some point set $h$ of $H^{\prime}, g$ is a point set of $G_{h}$. Let $g_{1}, g_{2}, g_{3}, \cdots$ denote the point sets of the collection $G$. There exists a sequence $P_{1}, P_{2}, P_{3}, \cdots$ such that for each positive integer $i, P_{i}$ is a point of $M$ which does not belong to any point set of the sequence $g_{1}, g_{2}+P_{1}, g_{3}+P_{2}, \cdots, g_{i}+P_{i-1} . \quad P_{1}+P_{2}+P_{3} \ldots$ is an infinite subset $K$ of $M$ such that no point set of $G$ contains infinitely many points of $K$.

THEOREM 1. If the hypothesis of the continuum is true, then there exists an uncountable subcollection of $H^{\prime}$ of the collection $H$ 
such that (1) if $h$ and $h^{\prime}$ are two point sets of $H^{\prime}$, no point set of $G_{h}$ contains infinitely many points of any one point set of $G_{h^{\prime}}$ and (2) if $K$ is an infinite subset of $M$, there exists a point set $h$ of $H^{\prime}$ such that some point set of the collection $G_{h}$ contains infinitely many points of $K$.

Proof. Let $W$ denote the collection of all infinite subsets of $M$. $W$ is equally numerous with the number interval $[0,1]$. Thus since no uncountable subset of $[0,1]$ is less numerous than $[0,1]$, there exists a meaning $P_{W}$ of the word precedes with respect to which $W$ is well ordered such that $h_{1}$ is the first point set of $W, h_{2}$ is the second point set of $W$, and no point set of $W$ is preceded by uncountably many point sets of $W$. There exists a meaning $P_{H}$ of the word preceded with respect to which the collection $H$ is well ordered such that $h_{1}$ is the first point set of $H$ and $h_{2}$ is the second point set of $H$.

It follows from Lemmas 1 and 3 that there exists a transformation $T$ of $W$ into a subcollection $H^{\prime}$ of $H$ such that (1) $T\left(w_{1}\right)=h_{1}$ and $T\left(w_{2}\right)=h_{2},(2)$ if $w$ is a point set of $W$ distinct from $w_{1}$ and $w_{2}$ such that for some point set $w^{\prime}$ of $W$ preceding $w$, some point set of the collection $G_{T\left(w^{\prime}\right)}$ contains infinitely many points of $w$, then for the first such point set $w^{\prime \prime}$ of $W$ in the $P_{W}$ sense, $T(w)=T\left(w^{\prime \prime}\right)$ and (3) if $w$ is a point set of $W$ distinct from $w_{1}$ and $w_{2}$ such that for each point set $w^{\prime}$ of $W$ preceding $w$, no point set of the collection $G_{T_{\left(w^{\prime}\right)}}$ contains infinitely many points of $w, T(w)$ is the first point set $h$ of the collection $H$ in the $P_{I I}$ sense such that $h$ contains infinitely many points of $w$ and for each point set $w^{\prime \prime}$ of $W$ that precedes $w$ in the $P_{W}$ sense, $h$ does not contain infinitely many points of any point set of the collection $G_{T\left(w^{\prime \prime}\right)}$.

It follows from Lemma 4 that $H^{\prime}$ is an uncountable subcollection of $H$. The collection $H^{\prime}$ fulfills the requirements of Theorem 1 .

THEOREM 2. If the hypothesis of the continuum is true, there exists a separable space satisfying Axions 0, 1, 2, 3, 4, 5, and 6 of [1] and containing a conditionally compact point set whose closure is not compact.

Proof. Let $M, G_{h}, h_{1}$, and $h_{2}$ be as previously defined. Let $H^{\prime}$ denote some collection of point sets containing $h_{1}$ and $h_{2}$ and satisfying conditions (1) and (2) of Theorem 1. Let $G$ denote the collection to which $g$ belongs if and only if $g$ is a point set of $G_{h}$ for some point set $h$ of the collection $H^{\prime}$.

Suppose $P$ is a point $(x, y)$ of $M$. Let $A_{P}$ and $B_{P}$ denote the endpoints of an interval $I$ such that (1) $A_{P}$ is above $B_{P}$, (2) $P$ is the 
midpoint of $I$, (3) the length of $I$ is $(x+y)^{-1}$, and (4) $I$ has slope -1 . For each number $k$ between 0 and 1 let $A_{P k}$ denote the point $X$ of the interval $P A_{P}$ such that the length of the interval $X P$ is the product of $k$ and the length of the interval $P A_{P}$. Let $B_{P k}$ denote point $X$ of the interval $P B_{P}$ such that $P$ is the midpoint of the sub-interval $X A_{P k}$ of the interval $A_{P} B_{P}$.

Suppose $g$ is a point set of $G$ and $k$ is a number between 0 and 1. Let $L_{g k}$ denote the point set to which the point $w$ belongs if and only if for some positive integer $i, w$ is either a point of the interval $A_{P_{i} k} A_{P_{i+1} k}$ or a point of the interval $B_{P_{i} k} B_{P_{i+1} k}$, where $P_{i}$ is the $i^{\text {th }}$ point of $g$. Let $L_{g 0}$ denote the point set to which the point $w$ belongs if and only if for some positive integer $i, w$ is a point of the interval $P_{i} P_{i+1}$, where $P_{i}$ is the $i^{\text {th }}$ point of $g$.

Suppose $g$ is a point set of $G, k$ is a number between 0 and 1 , and $n$ is a positive integer. Let $R_{\text {gkn }}$ denote the set to which $w$ belongs if and only if either (1) $w$ is $L_{g v}$, (2) for some number $c$ between 0 and $k, w$ is $L_{g c}$, or (3) $w$ is a point of $E$ which is separated from $(0,0)$ by the point set consisting of $L_{g k}$ and the interval $A_{P} B_{P}$ for the $n^{\text {th }}$ point $P$ of $g$.

Suppose $g$ is a point set of $G, k_{1}$ and $k_{2}$ are numbers between 0 and 1 , and $n$ is a positive integer. Let $R_{g k_{1} k_{2} n}$ denote the set to which $w$ belongs if and only if either (1) for some number $k$ between $k_{1}$ and $k_{2}, w$ is $L_{g k}$ or (2) $w$ is a point of $E$ which is separated from $(0,0)$ by the sum of $L_{g k_{1}}, L_{g k 2}$, and the sub-intervals $A_{P k_{1}} A_{P k_{2}}$ and $B_{P k 1} B_{P k_{2}}$ of the interval $A_{P} B_{P}$ for the $n^{\text {th }}$ point $P$ of $g$.

Let $\Sigma$ denote a space such that (1) $P$ is a point of $\Sigma$ if and only if either (a) $P$ is a point of $E$ or (b) for some point set $g$ of $G$ and some number $k$ between 0 and $1, P$ is either $L_{g k}$ or $L_{g 0}$ and (2) $R$ is a region in $\Sigma$ if and only if either (a) for some point $P$ in $E$ and some positive integer $n, R$ is the interior of a circle with center $P$ and radius $1 / n$, (b) $R$ is $R_{g k n}$ for some $g, k$, and $n$, or (c) $R$ is $R_{g k_{1} k_{2} n}$ for some $\mathrm{g}, k_{1}, k_{2}$, and $n$.

The set $S$ of all points of $\Sigma$ is the sum of two mutually exclusive point sets $E$ and $F$. If $P$ is a point of $F$, then $P$ is $L_{g k}$ for some $g$ and $k$ (including $k=0$ ); indeed, $P$ is a limit point of the infinite subset $A_{P_{1} k}+A_{P_{2} k}+A_{P_{3} k}+\cdots$ of $E$ (in case $k=0, A_{P_{j} k}=$ $P_{j}$ ) where $P_{1}+P_{2}+P_{3} \cdots=g$. Since each point of $F$ is a limit point of $E, S$ is separable. For each point set $g$ of $G$, let $R_{g}$ denote the ray in $\Sigma$ whose points are the points $L_{g k}$ for each nonnegative number $k$ less than 1 . If $P$ is a point of $R_{g}$ for some point set $g$ of $G$ and $g^{\prime}$ is a point set of $G$ distinct from $g$, no region containing $P$ contains a point of $R_{g^{\prime}}$.

It follows from Theorem 1 that if $K$ is an infinite subset of $M$, 
the set of all points $P$ of $E$ such that each coordinate of $P$ is a positive integer, then some point set $g$ of the collection $G$ contains infinitely many points of $K$. Thus in $\Sigma, L_{g 0}$ is a limit point of $K$ and $M$ is conditionally compact. $\bar{M}-M$ is the set of all points $L_{g 0}$ for all point sets $g$ of $G$. If $P$ is a point of $\bar{M}-M$ and $R$ is a region containing $P, R$ does not contain any point of $R_{g}$ for any point set $g$ of $G$ distinct from the one that converges to $P$. No point of $E$ is a limit point of $F$ and $\bar{M}-M$ is a subset of $F$, thus $\bar{M}-M$ has no limit point. Therefore $M$ is a conditionally compact point set whose closure is not compact.

\section{BIBLIOGRAPHY}

1. R. L. Moore, Foundations of point set theory, Amer. Math. Soc. Colloq. Publ. Vol. 13, rev. ed., Providence, R. I., 1962.

2. G. T. Whyburn Analytic topology, Amer. Math. Soc. Colloq. Publ. Vol. 28, rev. ed., Providence, R. I., 1963.

Received December 18, 1969. This paper is a part of the author's doctoral dissertation written at the University of Texas under the supervision of Professor R. L. Moore. Presented in part to the American Mathematical Society in Chicago, April 15, 1967, under a different title.

The University of TeXas

The UNIVERSity of MississipPI 



\section{PACIFIC JOURNAL OF MATHEMATICS}

\section{EDITORS}

\author{
H. SAMELSON \\ Stanford University \\ Stanford, California 94305

\section{Richard Pierce} \\ University of Washington \\ Seattle, Washington 98105
}

J. DugundJI

Department of Mathematics

University of Southern California

Los Angeles, California 90007

RichaRd ARENS

University of California

Los Angeles, California 90024

\section{ASSOCIATE EDITORS}
E. F. BECKENBACH
B. H. NeUMANN
F. WOLE
K. YoshidA

\section{SUPPORTING INSTITUTIONS}

\author{
UNIVERSITY OF BRITISH COLUMBIA \\ CALIFORNIA INSTITUTE OF TECHNOLOGY \\ UNIVERSITY OF CALIFORNIA \\ MONTANA STATE UNIVERSITY \\ UNIVERSITY OF NEVADA \\ NEW MEXICO STATE UNIVERSITY \\ OREGON STATE UNIVERSITY \\ UNIVERSITY OF OREGON \\ OSAKA UNIVERSITY \\ UNIVERSITY OF SOUTHERN CALIFORNIA
}

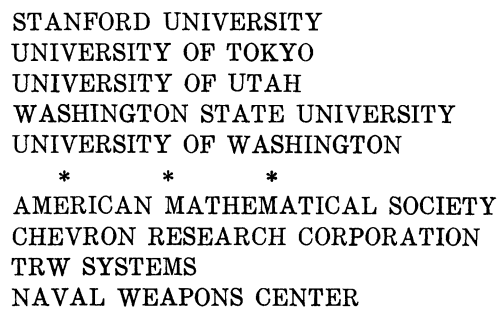

The Supporting Institutions listed above contribute to the cost of publication of this Journal, but they are not owners or publishers and have no responsibility for its content or policies.

Mathematical papers intended for publication in the Pacific Journal of Mathematics should be in typed form or offset-reproduced, (not dittoed), double spaced with large margins. Underline Greek letters in red, German in green, and script in blue. The first paragraph or two must be capable of being used separately as a synopsis of the entire paper. The editorial "we" must not be used in the synopsis, and items of the bibliography should not be cited there unless absolutely necessary, in which case they must be identified by author and Journal, rather than by item number. Manuscripts, in duplicate if possible, may be sent to any one of the four editors. Please classify according to the scheme of Math. Rev. Index to Vol. 39. All other communications to the editors should be addressed to the managing editor, Richard Arens, University of California, Los Angeles, California, 90024.

50 reprints are provided free for each article; additional copies may be obtained at cost in multiples of 50 .

The Pacific Journal of Mathematics is published monthly. Effective with Volume 16 the price per volume (3 numbers) is $\$ 8.00$; single issues, $\$ 3.00$. Special price for current issues to individual faculty members of supporting institutions and to individual members of the American Mathematical Society: $\$ 4.00$ per volume; single issues $\$ 1.50$. Back numbers are available.

Subscriptions, orders for back numbers, and changes of address should be sent to Pacific Journal of Mathematics, 103 Highland Boulevard, Berkeley, California, 94708.

PUBLISHED BY PACIFIC JOURNAL OF MATHEMATICS, A NON-PROFIT CORPORATION

Printed at Kokusai Bunken Insatsusha (International Academic Printing Co., Ltd.), 7-17, Fuj̣imi 2-chome, Chiyoda-ku, Tokyo, Japan. 


\section{Pacific Journal of Mathematics}

\section{Vol. 35, No. $2 \quad$ October, 1970}

Valentin Danilovich Belousov and Palaniappan L. Kannappan, Generalized Bol functional equation .................................... 259

Charles Morgan Biles, Gelfand and Wallman-type compactifications ........... 267

Louis Harvey Blake, A generalization of martingales and two consequent convergence theorems .................................... 279

Dennis K. Burke, On p-spaces and $w \Delta$-spaces..................... 285

John Ben Butler, Jr., Almost smooth perturbations of self-adjoint operators . . . . . . 297

Michael James Cambern, Isomorphisms of $C_{0}(Y)$ onto $C(X) \ldots \ldots \ldots \ldots \ldots . \ldots 307$

David Edwin Cook, A conditionally compact point set with noncompact closure ... 313

Timothy Edwin Cramer, Countable Boolean algebras as subalgebras and homomorphs .........................................

John R. Edwards and Stanley G. Wayment, A v-integral representation for linear operators on spaces of continuous functions with values in topological vector spaces.............................................

Mary Rodriguez Embry, Similarities involving normal operators on Hilbert

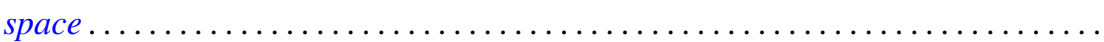

Lynn Harry Erbe, Oscillation theorems for second order linear differential

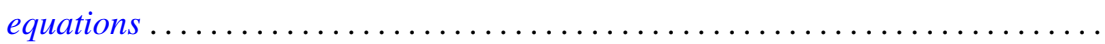

William James Firey, Local behaviour of area functions of convex bodies .......... Joe Wayne Fisher, The primary decomposition theory for modules ..............

Gerald Seymour Garfinkel, Generic splitting algebras for Pic ..................

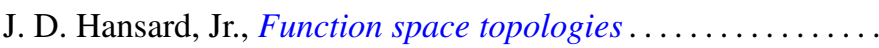

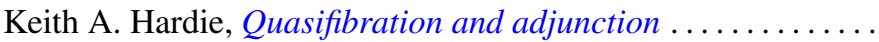

G. Hochschild, Coverings of pro-affine algebraic groups ...........

Gerald L. Itzkowitz, On nets of contractive maps in uniform spaces ..

381

389

399

417

Melven Robert Krom and Myren Laurance Krom, Groups with free nonabelian subgroups....................................

James Robert Kuttler, Upper and lower bounds for eigenvalues by finite differences ......................................

Dany Leviatan, A new approach to representation theory for convolution transforms . . .

Richard Beech Mansfield, Perfect subsets of definable sets of real numbers ...

Brenda MacGibbon, A necessary and sufficient condition for the embedding of a

Lindelof space in a Hausdorff $\mathscr{H} \sigma$ space ..................

David G. Mead and B. D. McLemore, Ritt's question on the Wronskian ....

Edward Yoshio Mikami, Focal points in a control problem .....

Paul G. Miller, Characterizing the distributions of three independent n-dimensional random variables, $X_{1}, X_{2}, X_{3}$, having analytic characteristic functions by the joint distribution of $\left(X_{1}+X_{3}, X_{2}+X_{3}\right)$. . .

P. Rosenthal, On the Bergman integral operator for an elliptic partial differential equation with a singular coefficient....

Douglas B. Smith, On the number of finitely generated $O$-group 